\title{
Multicomponent alloy solidification: Phase-field modeling and simulations
}

\author{
Britta Nestler \\ Department of Computer Science, Karlsruhe University of Applied Sciences, Moltkestrasse 30, D-76133 Karlsruhe, Germany \\ Harald Garcke and Björn Stinner \\ NWF I, Mathematik, University of Regensburg, 93040 Regensburg, Germany \\ (Received 14 May 2004; revised manuscript received 6 December 2004; published 27 April 2005)
}

\begin{abstract}
A general formulation of phase-field models for nonisothermal solidification in multicomponent and multiphase alloy systems is derived from an entropy functional in a thermodynamically consistent way. General expressions for the free energy densities, for multicomponent diffusion coefficients, and for both weak and faceted types of surface energy and kinetic anisotropy are possible. A three-dimensional simulator is developed to show the capability of the model to describe phase transitions, complex microstructure formation, and grain growth in polycrystalline textures.
\end{abstract}

DOI: 10.1103/PhysRevE.71.041609 PACS number(s): 81.10. $-\mathrm{h}, 05.10 .-\mathrm{a}$, 81.30. $-\mathrm{t}$, 68.35. $-\mathrm{p}$

\section{INTRODUCTION}

Alloy systems with multiple components are an important class of materials, in particular for technical applications and processes. The microstructure formation of a material plays a central role for a broad range of mechanical properties and, hence, for the quality and the durability of the material. Aiming for a continuous optimization of materials properties, the study of pattern formation during alloy solidification has been a focus of many experimental and, recently, also of computational work. Since the microstructure characteristics are a result of the process conditions used during production, the analysis of the fundamental correlation between the processing pathway and the microstructure is of fundamental importance. Multiple components in alloys are combined with the appearance of multiple phases leading to complex phase diagrams, various phase transformations, and different types of solidification. Modeling and numerical simulations aim to predict microstructure evolution in multicomponent alloys in order to virtually design materials. However, the great number of material parameters and physical variables involved in systems yields a complexity that remains a big challenge for future work. In particular, the gain of statistically meaningful data from computations requires simulations in sufficiently large domains with a tremendous need of memory and computing time resources. To treat complex systems, high-performance computing, parallelization, and optimized algorithms including adaptive mesh generators are mandatory.

The phase-field method has become an important tool for tackling free boundary problems such as grain boundary motion $[1,2]$ and for simulating crystal growth, solidification, and pattern formation phenomena in alloys [3-15]. The advantage of the phase-field method lies in the formulation of diffuse interfaces of a finite thickness. Explicit front tracking is avoided by using smooth continuous variables locating the grain and phase boundaries. By asymptotic expansions for vanishing interface thickness, it can be shown that classical sharp interface models including physical laws at interfaces and multiple junctions are recovered [16,17]. Since phasefield models can be derived on the basis of classical irrevers- ible thermodynamics $[18-20]$, they can be applied to processes close to thermodynamical equilibrium-i.e., at relatively small driving forces. Extensions of the phase-field approach to describe strongly nonequilibrium solidification, solute trapping, and solute drag effects at large driving forces are discussed in $[21,22]$. The scaling problem of quantitatively modeling the low-growth-rate regime where the microstructure is typically several orders of magnitude larger than the microscopic capillary length has been overcome by considering a so-called thin interface limit of the phase-field model [23-27].

The purpose of this paper is to extend the advances of the phase-field approach to model general nonisothermal multicomponent multiphase solidification in situations close to local thermodynamical equilibrium and to generalize former models $[20,28]$. The underlying general formulation of founded on an entropy functional is given in Sec. II. Explicit expressions for the free energy densities of the bulk phases and interfaces are discussed. A method is described how the artifical appearance of foreign phase contributions at a twophase boundary can be avoided. Formulations defining the bulk, interdiffusion, and interfacial diffusion coefficients as well as different types of surface energies and kinetic anisotropies are presented. In particular, an expression of crystalline (faceted) anisotropy is given that can be used for modeling general crystal shapes with an arbitrary number of edges and corners in three spatial dimensions. The essential ingredients of the phase-field model are summarized here, the numerical method for solving the governing equations is briefly explained, and examples of possible applications to numerically simulate moving grain and phase boundaries in binary and ternary alloys are given in Sec. III. The simulation results are intended to illustratively show the potential of the phase-field model in computing and numerically analyzing complex pattern formations in multicomponent alloys.

\section{PHASE-FIELD MODEL OF MULTICOMPONENT SOLIDIFICATION}

The phase-field model is formulated for a general class of multicomponent and multiphase alloy systems consisting of 
$K$ components and $N$ different phases in a domain $\Omega$. It is assumed that the system is in mechanical equilibrium and, for simplicity, that the pressure and mass density are constant. The concentrations of the components are represented by a vector $\boldsymbol{c}(\vec{x}, t)=\left(c_{1}(\vec{x}, t), \ldots, c_{K}(\vec{x}, t)\right)$. Similarly, the phase fractions are described by a vector-valued order parameter $\boldsymbol{\phi}(\vec{x}, t)=\left(\phi_{1}(\vec{x}, t), \ldots, \phi_{N}(\vec{x}, t)\right)$. The variable $\phi_{\alpha}(\vec{x}, t)$ denotes the local fraction of phase $\alpha$. It is required that the concentrations of the components and the phase-field variables fulfill the constraints

$$
\sum_{i=1}^{K} c_{i}=1 \text { and } \sum_{\alpha=1}^{N} \phi_{\alpha}=1
$$

The physical effects occurring during solidification such as heat and mass transfer, the release of latent heat, the GibbsThomson relation, and interface kinetics are obtained on the basis of an entropy functional $S(e, c, \boldsymbol{\phi})$ of the form

$$
S(e, \boldsymbol{c}, \boldsymbol{\phi})=\int_{\Omega}\left[s(e, \boldsymbol{c}, \boldsymbol{\phi})-\left(\varepsilon a(\boldsymbol{\phi}, \boldsymbol{\nabla} \boldsymbol{\phi})+\frac{1}{\varepsilon} w(\boldsymbol{\phi})\right)\right] d x,
$$

depending on the internal energy $e$, the concentrations $c_{i}, i$ $=1, \ldots, K$, and the phase fields $\phi_{\alpha}, \alpha=1, \ldots, N$. The first term in the entropy functional $s(e, \boldsymbol{c}, \boldsymbol{\phi})$ is a bulk entropy density. The second and third summands $a(\boldsymbol{\phi}, \boldsymbol{\nabla} \boldsymbol{\phi})$ and $w(\boldsymbol{\phi})$ model surface entropy densities taking into account the thermodynamics of the interfaces. As typical in diffuse interface models, $\varepsilon$ is a small-length scale parameter related to the thickness of the diffuse interface.

Knowing the free energy densities of the pure phases $f_{\alpha}(T, \boldsymbol{c})$, the total free energy $f(T, \boldsymbol{c}, \boldsymbol{\phi})$ is obtained as a suitable interpolation of the $f_{\alpha}$. The Gibbs relation reads (observe that due to the assumptions the Gibbs free energy and the Helmholtz free energy coincide up to a constant)

$$
\begin{aligned}
d f & =f_{, T} d T+\sum_{i} f_{, c_{i}} d c_{i}+\sum_{\alpha} f_{, \phi_{\alpha}} d \phi_{\alpha} \\
& =-s d T+\sum_{i} \mu_{i} d c_{i}+\sum_{\alpha} r_{\alpha} d \phi_{\alpha},
\end{aligned}
$$

where $T$ is the temperature, $s=-f_{, T}$ is the entropy density, $\mu_{i}=f_{, c_{i}}$ are the chemical potentials, and $r_{\alpha}=f_{, \phi_{\alpha}}$ are potentials due to the appearance of different phases. Here, $f_{, X}$ denotes the partial derivative of the free energy $f$ with respect to $X$. The internal energy density is given by $e=f+s T$. From this relation it can be derived that $s_{, e}=1 / T$ and $s_{, c_{i}}=-\mu_{i} / T$.

Through the free energies $f_{\alpha}$, a general class of phase diagrams for multiphase multicomponent alloy systems can be incorporated into the phase-field model. The model allows for systems with general free energies $f_{\alpha}(T, \boldsymbol{c})$ being convex in $c$ and concave in $T$. Choosing the liquid phase to be the last component $\phi_{N}$ of the phase-field vector $\phi$, an ideal solution formulation of the bulk free energy density $f_{i d}(T, \boldsymbol{c}, \boldsymbol{\phi})$ reads

$$
\begin{aligned}
f_{i d}(T, \boldsymbol{c}, \boldsymbol{\phi}):= & \sum_{\alpha=1}^{N} \sum_{i=1}^{K}\left(c_{i} L_{i}^{\alpha} \frac{T-T_{i}^{\alpha}}{T_{i}^{\alpha}} h\left(\phi_{\alpha}\right)\right) \\
& +\sum_{i=1}^{K}\left(\frac{R_{g}}{v_{m}} T c_{i} \ln \left(c_{i}\right)\right)-c_{v} T \ln \left(\frac{T}{T_{M}}\right),
\end{aligned}
$$

with $L_{i}^{N}=0$ and $L_{i}^{\alpha}, i=1, \ldots, K, \alpha=1, \ldots, N-1$, being the latent heat per unit volume of the phase transition from phase $\alpha$ to the liquid phase and of pure component $i$. Furthermore, $T_{i}^{\alpha}, i=1, \ldots, K, \alpha=1, \ldots, N-1$ is the melting temperature of the $i$ th component in phase $\alpha$, and $T_{M}$ is a reference temperature. $c_{v}$, the specific heat and $v_{m}$, the molar volume, are assumed to be constant, and $R_{g}$ is the gas constant. With a suitable choice of the function $h(\boldsymbol{\phi})$ satisfying $h(0)=0$ and $h(1)=1$ - e.g., $h\left(\phi_{\alpha}\right)=\phi_{\alpha}$ or $h\left(\phi_{\alpha}\right)=\phi_{\alpha}^{2}\left(3-2 \phi_{\alpha}\right)$-the free energy density $f$ is an interpolation of the individual free energy densities $f_{\alpha}$. A more general expression for alloys is the Redlich-Kister-Muggianu model of subregular solution,

$$
f_{s r}=f_{i d}+\sum_{i=1}^{K} \sum_{j=1}^{K} c_{i} c_{j} \sum_{\nu=0}^{M} M_{i j}^{(\nu)}\left(c_{i}-c_{j}\right)^{\nu}
$$

with binary interaction coefficients $M_{i j}^{(\nu)}$ depending on the parameter $\nu$. For $M=0$, the Redlich-Kister-Muggianu ansatz takes the form of a regular solution model. In most applications, in particular to metallic systems, $M$ takes a maximum value of 2 . A ternary term $\sim c_{i} c_{j} c_{k}$ can be added to describe the excess free energy.

The gradient entropies $a(\boldsymbol{\phi}, \boldsymbol{\nabla} \boldsymbol{\phi})$ can be expressed in terms of a generalized antisymmetric gradient vector $q_{\alpha \beta}$ $=\phi_{\alpha} \nabla \phi_{\beta}-\phi_{\beta} \nabla \phi_{\alpha}$ by

$$
a(\boldsymbol{\phi}, \boldsymbol{\nabla} \boldsymbol{\phi})=\sum_{\alpha<\beta} A_{\alpha \beta}\left(q_{\alpha \beta}\right)=\sum_{\alpha<\beta} \frac{\gamma_{\alpha \beta}}{m_{\alpha \beta}}\left[a_{\alpha \beta}\left(q_{\alpha \beta}\right)\right]^{2}\left|q_{\alpha \beta}\right|^{2},
$$

where $\gamma_{\alpha \beta}$ represent surface entropy densities and $m_{\alpha \beta}$ are mobility coefficients. The formulation using the generalized gradient vector $q_{\alpha \beta}$ allows us to distinguish the physics of each phase (or grain) boundary by providing enough degrees of freedom. The anisotropy of the surface entropy density is modeled by the factor $\left[a_{\alpha \beta}\left(q_{\alpha \beta}\right)\right]^{2}$ depending on the orientation of the interface. The factors are homogeneous of degree 0 and hence $A_{\alpha \beta}\left(q_{\alpha \beta}\right)$ is homogeneous of degree 2. Phase boundaries with isotropic surface entropies are realized by $a_{\alpha \beta}\left(q_{\alpha \beta}\right)=1$. Weakly anisotropic crystals with an underlying cubic symmetry can be modeled by the expression

$$
a_{\alpha \beta}\left(q_{\alpha \beta}\right)=1-\delta_{\alpha \beta}\left(3 \mp 4 \frac{\left|q_{\alpha \beta}\right|_{4}^{4}}{\left|q_{\alpha \beta}\right|^{4}}\right),
$$

with $|q|_{4}^{4}=\sum_{i=1}^{d}\left(q_{i}^{4}\right)$ and $|q|^{4}=\left[\sum_{i=1}^{d}\left(q_{i}^{2}\right)\right]^{2}$. The strength of the anisotropy of an $\alpha / \beta$ phase or grain boundary is given by the parameter $\delta_{\alpha \beta}$. For a strongly anisotropic crystal of faceted type, we define 


$$
a_{\alpha \beta}\left(q_{\alpha \beta}\right)=\max _{1 \leqslant k \leqslant n_{\alpha \beta}}\left\{\frac{q_{\alpha \beta}}{\left|q_{\alpha \beta}\right|} \eta_{\alpha \beta}^{k}\right\},
$$

where $\eta_{\alpha \beta}^{k}, k=1, \ldots, n_{\alpha \beta}$, are the $n_{\alpha \beta}$ corners of the Wulff shape of the $\alpha-\beta$ transition. Equation (2) allows us to model arbitrary crystal shapes with $n_{\alpha \beta}$ corners.

The interfacial free entropy density $w(\boldsymbol{\phi})$ might be of multiwell type $w_{s t}(\boldsymbol{\phi})$ or of a multiobstacle type $w_{o b}(\phi)$. Suitable expressions of these potentials can be formulated as extensions of the double well or obstacle potential for solidliquid phase-field models:

$$
\begin{gathered}
w_{s t}(\boldsymbol{\phi})=9 \sum_{\alpha<\beta} m_{\alpha \beta} \gamma_{\alpha \beta} \phi_{\alpha}^{2} \phi_{\beta}^{2}, \\
w_{o b}(\boldsymbol{\phi})=\frac{16}{\pi^{2}} \sum_{\alpha<\beta} m_{\alpha \beta} \gamma_{\alpha \beta} \phi_{\alpha} \phi_{\beta} .
\end{gathered}
$$

In the obstacle case, we set $w_{o b}(\boldsymbol{\phi})=\infty$ if $\phi$ is not on the Gibbs simplex $G=\left\{\boldsymbol{\phi} \in \mathbb{R}^{N}: \Sigma_{\alpha} \boldsymbol{\phi}_{\alpha}=1, \boldsymbol{\phi}_{\alpha} \geqslant 0\right\}$. From a computational point of view, an advantage of using obstacle-type potentials for numerical simulations is that the partial differential equations for the phase fields $\phi_{\alpha}, \alpha=1, \ldots, N$, only need to be solved in a finite region of the diffuse interface layer.

The relation between the surface entropy $\bar{\gamma}_{\alpha \beta}(\nu)$ of an $\alpha-\beta$ phase boundary with orientation $\nu$ and the interface terms is given by

$$
\bar{\gamma}_{\alpha \beta}(\nu)=2 \inf _{p} \int_{-1}^{1} \sqrt{a\left(p, p^{\prime} \otimes \nu\right) w(p)} d y,
$$

where the infimum is taken over all Lipschitz continuous functions $p:[-1,1] \rightarrow G$ with $p(-1)=e_{\alpha}, p(1)=e_{\beta}$ (see [2] for the details). The calibration properties of the phase-field parameters $\gamma_{\alpha \beta}$ with respect to experimentally given data are optimal if $\bar{\gamma}_{\alpha \beta}=\gamma_{\alpha \beta}$. But in general, the minimizer is difficult to determine, because it does not follow the edge of the Gibbs simplex $G$ which results in the presence of phase fields different from $\phi_{\alpha}$ and $\phi_{\beta}$ in the transition region. To avoid such third-phase contributions at a two-phase interface, additional terms of third order $\sim \phi_{\alpha} \phi_{\beta} \phi_{\delta}$ can be added to the multiobstacle potential-i.e.,

$$
\tilde{w}_{o b}(\boldsymbol{\phi})=w_{o b}(\boldsymbol{\phi})+\sum_{\alpha<\beta<\delta} \gamma_{\alpha \beta \delta} \phi_{\alpha} \phi_{\beta} \phi_{\delta}
$$

A sufficient condition for choosing the parameter $\gamma_{\alpha \beta \delta}$ in order to optimize the calibration properties is

$$
\gamma_{\alpha \beta \delta} \geqslant \frac{96 \gamma_{i j} m_{i j}}{\pi^{2}}, \quad \text { for all }(i, j) \in\{(\alpha, \beta),(\beta, \delta),(\delta, \alpha)\} .
$$

Alternatively smooth multiwell potentials of the form

$$
w(\boldsymbol{\phi})=w_{s t}(\boldsymbol{\phi})+72 \sum_{\alpha<\beta}\left(\gamma_{\alpha \beta} m_{\alpha \beta} \phi_{\alpha}^{2} \phi_{\beta}^{2} \sum_{\delta \neq \alpha, \beta} \phi_{\delta}\right)
$$

so far yield very promising results to avoid the appearance of artificial third-phase contributions. In spite of the fifth-order terms, the multiwell structure is attained on the Gibbs simplex $G$, but because of numerical errors in the simulations, additional terms outside of $G$ must be added so that the potential is bounded from below by zero. The above potentials will be studied in more detail in a forthcoming paper [29]. For further approaches we refer to [30] and [31] (which is based on ideas of [32]).

The governing set of equations follows from conservation laws for the internal energy $e$ and the concentrations $c_{i}, i$ $=1, \ldots, K$, coupled to a gradient flow for the nonconserved phase-field variables $\phi_{\alpha}, \alpha=1, \ldots, N$. The equations are derived by variational differentiation of the entropy functional $S(e, \boldsymbol{c}, \boldsymbol{\phi})$, ensuring energy and mass conservation and the increase of total entropy. They read

$$
\begin{aligned}
& \frac{\partial e}{\partial t}=-\boldsymbol{\nabla} \cdot\left[L_{00} \boldsymbol{\nabla} \frac{1}{T}+\sum_{j=1}^{K} L_{0 j} \boldsymbol{\nabla}\left(\frac{-\mu_{j}}{T}\right)\right], \\
& \frac{\partial c_{i}}{\partial t}=-\boldsymbol{\nabla} \cdot\left[L_{i 0} \boldsymbol{\nabla} \frac{1}{T}+\sum_{j=1}^{K} L_{i j} \boldsymbol{\nabla}\left(\frac{-\mu_{j}}{T}\right)\right], \\
& \tau \varepsilon \frac{\partial \phi_{\alpha}}{\partial t}=\varepsilon\left[\nabla \cdot a_{, \nabla \phi_{\alpha}}(\boldsymbol{\phi}, \boldsymbol{\nabla} \boldsymbol{\phi})-a_{, \phi_{\alpha}}(\boldsymbol{\phi}, \boldsymbol{\nabla} \boldsymbol{\phi})\right] \\
& -\frac{w_{, \phi_{\alpha}}(\boldsymbol{\phi})}{\varepsilon}-\frac{f_{, \phi_{\alpha}}(T, \boldsymbol{c}, \boldsymbol{\phi})}{T}-\lambda,
\end{aligned}
$$

where $\boldsymbol{\nabla} \cdot(\cdots)$ denotes the divergence of the term in the brackets. $a_{, \phi_{\alpha}}, w_{, \phi_{\alpha}}, f_{, \phi_{\alpha}}$, and $a_{, \nabla \phi_{\alpha}}$ are the derivatives of the energy contributions with respect to $\phi_{\alpha}$ and $\nabla \phi_{\alpha}$, respectively. The parameter $\lambda$ in Eq. (6) is a Lagrange multiplier garantueeing that the constraint $\Sigma_{\alpha=1}^{N} \phi_{\alpha}=1$ is preservedi.e.,

$$
\begin{aligned}
\lambda= & \frac{1}{N} \sum_{\alpha=1}^{N}\left[\varepsilon\left[\boldsymbol{\nabla} \cdot a_{, \boldsymbol{\nabla} \phi_{\alpha}}(\boldsymbol{\phi}, \boldsymbol{\nabla} \boldsymbol{\phi})-a_{, \phi_{\alpha}}(\boldsymbol{\phi}, \boldsymbol{\nabla} \boldsymbol{\phi})\right]\right. \\
& \left.-\frac{w_{, \phi_{\alpha}}(\boldsymbol{\phi})}{\varepsilon}-\frac{f_{, \phi_{\alpha}}(T, \boldsymbol{c}, \boldsymbol{\phi})}{T}\right] .
\end{aligned}
$$

In referring to nonequilibrium thermodynamics, we postulate the fluxes for the conserved quantities to be linear combinations of the thermodynamical driving forces $\boldsymbol{\nabla} \delta S / \delta e=\boldsymbol{\nabla}(1 / T)$ and $\boldsymbol{\nabla} \delta S / \delta c_{i}=\boldsymbol{\nabla}\left(-\mu_{i} / T\right)$.

To fulfill the constraint $\sum_{i=1}^{K} c_{i}=1$ during the evolution, it is required that $\sum_{i=1}^{K} L_{i j}=0, j=0, \ldots, K$. Further, it is assumed that the matrix $L=\left(L_{i j}\right)_{i, j}$ is positive semidefinite and symmetric according to the Onsager relations. It can be shown that this condition leads to a local entropy inequality ensuring non-negative local entropy production. The mobility coefficients $\left(L_{i j}\right)_{i, j=0, \ldots, K}$ are allowed to depend on $T, \boldsymbol{c}$, and $\boldsymbol{\phi}$. Given some heat and mass diffusion coefficients $k$ $=k(T, \boldsymbol{c}, \boldsymbol{\phi})$ and $D_{i}=D_{i}(T, \boldsymbol{c}, \boldsymbol{\phi})$, the $L_{i j}$ can be related to them by

$$
L_{j i}=L_{i j}=\frac{v_{m}}{R_{g}} D_{i} c_{i}\left(\delta_{i j}-\frac{D_{j} c_{j}}{\sum_{k=1}^{K} D_{k} c_{k}}\right)
$$

for $i, j=1, \ldots, K$ and then recursively, 


$$
\begin{gathered}
L_{0 j}=-\frac{v_{m}}{R_{g}} \sum_{\alpha=1}^{N} \sum_{i=1}^{K} L_{j i} h\left(\phi_{\alpha}\right) L_{i}^{\alpha}, \\
L_{00}=k T^{2}+\frac{v_{m}}{R_{g}} \sum_{\alpha, \beta}^{N, N} \sum_{i, j}^{K, K} h\left(\phi_{\alpha}\right) L_{i}^{\alpha} L_{j i} h\left(\phi_{\beta}\right) L_{j}^{\beta},
\end{gathered}
$$

where $\delta_{i j}$ denotes the Kronecker delta and $L_{i}^{\alpha}$ are the latent heats of fusion. The formulation in Eqs. (7)-(9) takes bulk diffusion effects including interdiffusion coefficients into account. The dependence of the mass and heat diffusion coefficients on $\boldsymbol{\phi}$ can be realized by, e.g., linear expansions. To also consider enhanced diffusion in the interfacial region of phase or grain boundaries, additional terms proportional to $\phi_{\alpha} \phi_{\beta}$ with interfacial diffusion coefficients $D_{i}^{\alpha \beta}\left(T, c, q_{\alpha \beta}\right)$ need to be added. Altogether, we suggest

$$
D_{i}=\sum_{\alpha=1}^{N} D_{i}^{\alpha}(T, \boldsymbol{c}) \phi_{\alpha}+\frac{1}{\varepsilon} \sum_{\alpha<\beta} D_{i}^{\alpha \beta}\left(T, \boldsymbol{c}, q_{\alpha \beta}\right) \phi_{\alpha} \phi_{\beta},
$$

i.e., in particular that the diffusion coefficients in Eq. (7) can be anisotropic. The quantity $\tau=\tau(\boldsymbol{\phi}, \boldsymbol{\nabla} \boldsymbol{\phi})$ in Eq. (6) models an anisotropic kinetic coefficient of the form

$$
\tau(\boldsymbol{\phi}, \nabla \boldsymbol{\phi})=\tau_{0}+\sum_{\alpha<\beta} B_{\alpha \beta}\left(q_{\alpha \beta}\right),
$$

with $B_{\alpha \beta}\left(q_{\alpha \beta}\right)=0$ if $q_{\alpha \beta}=0$. Possible choices are

$$
B_{\alpha \beta}=\tau_{\alpha \beta}^{0}\left[1+\zeta_{\alpha \beta}\left(3 \pm 4 \frac{\left|q_{\alpha \beta}\right|_{4}^{4}}{\left|q_{\alpha \beta}\right|^{4}}\right)\right]-\tau_{0}
$$

or

$$
B_{\alpha \beta}=\tau_{\alpha \beta}^{0} \max _{1 \leqslant k \leqslant r_{\alpha \beta}}\left\{\frac{q_{\alpha \beta}}{\left|q_{\alpha \beta}\right|} \xi_{\alpha \beta}^{k}\right\}-\tau_{0},
$$

if $q_{\alpha \beta} \neq 0$ for weakly cubic [Eq. (10)] or strongly faceted [Eq. (11)] kinetic anisotropies with $r_{\alpha \beta}$ corners $\xi_{\alpha \beta}^{k}$. Here $\zeta_{\alpha \beta}$ determines the strength of the kinetic anisotropy similar to $\delta_{\alpha \beta}$ in Eq. (1) for the surface energy anisotropy. Systems with isotropic kinetics are realized by setting $\zeta_{\alpha \beta}=0$.

\section{SIMULATIONS OF SOLIDIFYING GROWTH STRUCTURES IN BINARY AND TERNARY SYSTEMS}

To compute microstructure formation, a threedimensional (3D) parallel simulator has been developed to numerically solve the set of equations for the internal energy, the concentrations, and the phase fields [Eqs. (4)-(6)]. The solving method is based on an explicit finite- difference discretization on a rectangular mesh. The following strategies to optimize the efficiency of the numerical algorithm with respect to computation time and memory usage are applied: An obstacle- type potential $w(\boldsymbol{\phi})$ as in Eq. (3) is used in the computations providing the advantage of solving the phasefield equations only in the region of the phase or grain boundaries-i.e., in the finite diffuse interface region of a width of approximately 10 numerical cells. This reduces the computational effort with respect to the phase-field equations
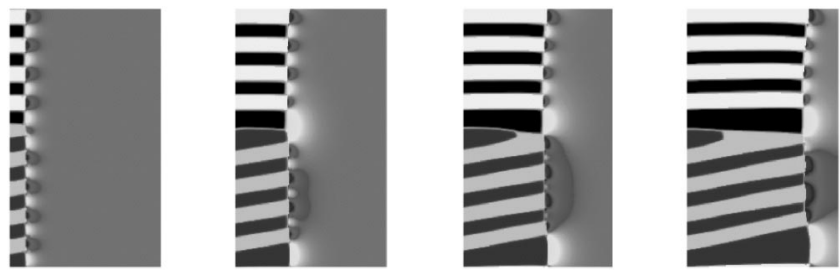

FIG. 1. Growth of two eutectic grains (white and black and light and dark grey) of a binary $A-B$ alloy with different crystal orientations into an isothermally undercooled melt (continuous grey scale) at four time steps.

by one spatial dimension. Three different time steps are chosen to solve the three types of equations [Eqs. (4)-(6)]. Similarly, three different spatial meshes are used to determine the internal energy, the concentration, and the phase-field equations. The values at intermediate grid points are interpolated from the coarsener mesh. Furthermore, a parallelization of the numerical algorithm is realized dividing the 3D computational domain into partial sublayers. The subdomains are computed on a Linux cluster of dual processor machines using a combination of two cooperating techniques: MPI (Message Passing Interface, standard library for implementing distributed parallelism) and OpenMP (Open Multi Processing, library for implementing shared memory parallelism).

In the following, microstructure simulations of binary and ternary phase transformation processes are shown to illustrate the wide variety of realistic growth structures and morphologies in multicomponent multiphase systems that can be described and investigated by the new phase-field model. Fields of applications are eutectic grain boundary formations and structure evolutions in ternary systems [33] as well as eutectic colony growth involving ternary impurity effects [34] which will be shown by the following examples, but also multicomponent dendritic and eutectic crystal growth can be simulated.

To perform the simulations in Figs. 1-4 we considered a

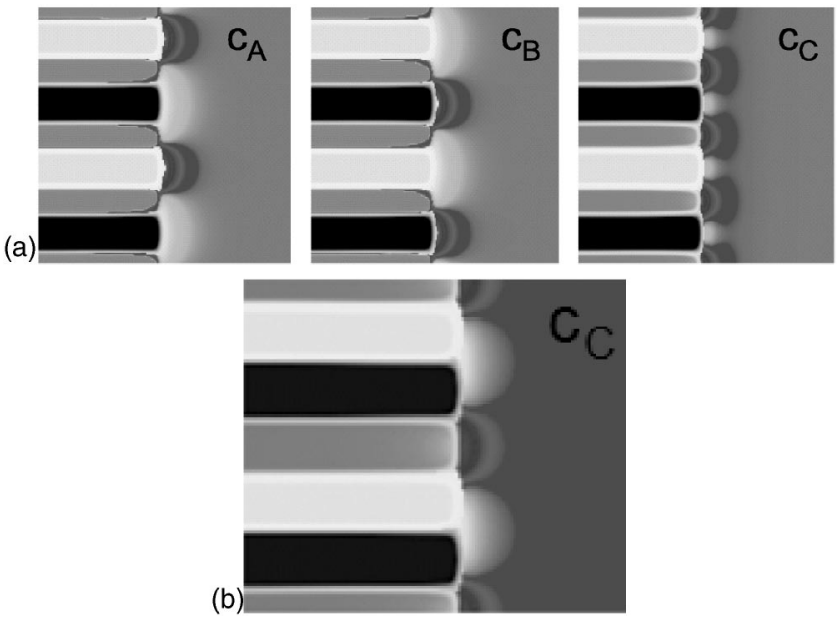

FIG. 2. (a) Concentration fields $c_{A}, c_{B}$, and $c_{C}$ of a ternary eutectic lamellar solidification front with a solid phase configuration $(\alpha|\beta| \alpha|\gamma| \cdots)$. (b) Concentration field $c_{C}$ for a phase sequence $(\alpha|\beta| \gamma \mid \cdots)$. 

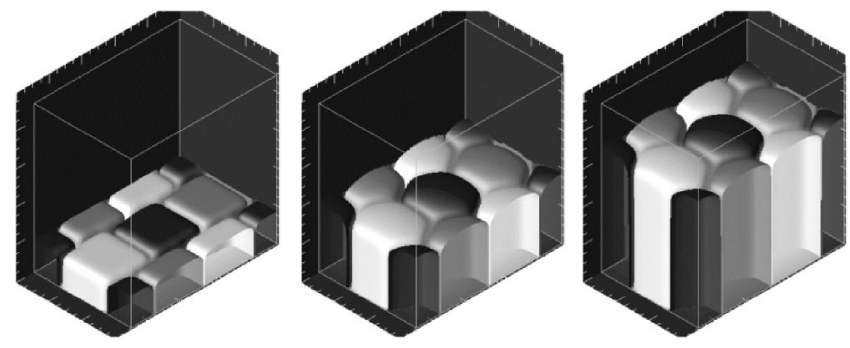

FIG. 3. Formation of a 3D hexagonal rodlike structure in a ternary eutectic system with isotropic surface energies and three different solid phases $\alpha, \beta$, and $\gamma$.

ternary eutectic model alloy system of three components $A$, $B$, and $C(i=1, \ldots, 3)$, three solid phases $\alpha, \beta$, and $\gamma(\alpha$ $=1, \ldots, 3)$, and one liquid phase $\boldsymbol{L}(\alpha=4)$. We nondimensionalized the model equations [Eqs. (4)-(6)] and, for initialization of the computations, we chose the following parameter set: Equal grid spacings for the two or three coordinates at a value $\Delta x=0.01$, mobility coefficients $m_{\alpha \beta}=0.1$, a diffusive interface thickness $\epsilon=0.05$, surface entropy densities $\gamma_{\alpha \beta}=0.001$, an isotropic kinetic coefficient $\tau=0.2$, zero diffusion in the solid phases, $D_{i=1, \ldots, 3}^{\alpha, \beta, \gamma}=0.0$, and diffusion coefficients in the liquid, $D_{i=1, \ldots, 3}^{L}=0.01$. Further, we constructed a completely symmetric phase diagram with dimensionless data for the latent heats of fusion, $L_{i}^{\alpha}$, and for the melting temperatures $T_{i}^{\alpha}$ :

$$
\begin{aligned}
\left(L_{i}^{\alpha}\right)_{\alpha=1, \ldots, 4}^{i=1, \ldots, 3} & =\left(\begin{array}{llll}
1.47 & 1.00 & 1.00 & 0.00 \\
1.00 & 1.47 & 1.00 & 0.00 \\
1.00 & 1.00 & 1.47 & 0.00
\end{array}\right), \\
\left(T_{i}^{\alpha}\right)_{\alpha=1, \ldots, 4}^{i=1, \ldots, 3} & =\left(\begin{array}{llll}
1.50 & 0.50 & 0.50 & 0.00 \\
0.50 & 1.50 & 0.50 & 0.00 \\
0.50 & 0.50 & 1.50 & 0.00
\end{array}\right),
\end{aligned}
$$

where $\alpha=4$ is assumed to be the liquid phase $L$. As a result, the phase fractions of the three solid phases at the ternary eutectic temperature are equal. Further, we considered the solidification process under the condition of isothermally undercooled melts.

In Fig. 1, the formation of two eutectic grains in the binary $A-B$ "edge" system of initial composition $\left(c_{A}, c_{B}, c_{C}\right)$ $=(0.5,0.5,0.0)$ has been simulated in a $2 \mathrm{D}$ domain of 270 $\times 540$ grid points. The simulation involves pattern formation on different length scales. On a larger scale, grains with different orientations due to anisotropy of the surface entropy densities $\gamma_{\alpha \beta}$ grow and form a eutectic grain boundary. To include anisotropic effects, we used the faceted formulation of Eq. (2) for a cubic crystal symmetry and defined two sets of four corners for the upper and for the lower grain, whereas the corners of the lower grain are rotated by $10^{\circ}$ with respect to the growth direction. On a smaller scale, a lamellar eutectic substructure solidifies: Below a critical eutectic temperature $T_{e}$ (here $T_{e}=1.0$ ), a parent liquid phase $L$ transforms into two solid phases $\alpha$ and $\beta$ in a binary eutectic reaction: $L$ $\rightarrow \alpha+\beta$. The white and light grey colored regions as well as the black and dark grey colored regions represent the same

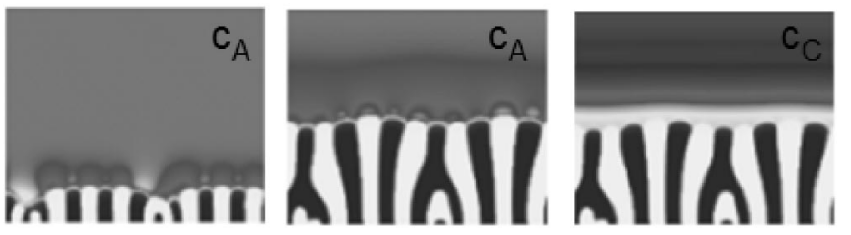

FIG. 4. Simulation of lamellar eutectic growth in a ternary system with an impurity component $c_{C}$ : The concentration profile of the main component $c_{A}$ in melt is shown in the left and centered images for two time steps. The ternary impurity $c_{C}$ is pushed ahead of the growing eutectic front so that concentration-enriched zones of component $c_{C}$ can be observed at the solid-liquid interface in the right image.

solid phases—namely, $\alpha$ and $\beta$-with just a different orientation. The result illustrates the capability of the model to distinguish several phases and grains at the same time. The images visualize the phase evolution and the concentration profile of the alloy component $B$ in the liquid ahead of the growing solid phases at different time steps. Concentration depleted zones occur in dark grey and concentration enriched zones appear in light grey.

Depending on the position in the phase diagram, ternary alloy solidification may involve phase changes of four different phases and diffusion of three alloy components $A, B$, and $C$. At the ternary eutectic composition, three solid phases grow into an undercooled melt via the reaction $L \rightarrow \alpha+\beta$ $+\gamma$. While simultaneously growing, the solid phases mutually enhance each other's growth conditions as they reject opposite components of the alloy into the liquid. We have set an equal initial composition vector of $\left(c_{A}, c_{B}, c_{C}\right)$ $=(0 . \overline{3}, 0 . \overline{3}, 0 . \overline{3})$. For isotropic phases, this leads to very regular lamellar structures as those in Figs. 2(a) and 2(b). The three images in (a) display the concentration fields of the three components $A, B$, and $C$ in front of the growing eutectic lamellae with a phase sequence of $\left.\alpha|\beta| \alpha|\gamma| \alpha\right|^{\cdots}$ at the same intermediate time step. It can be observed that the white $\alpha$ phase consumes component $A$ from the melt and pushes components $B$ and $C$ into the melt. The respective process happens for the two other solids $\beta$ and $\gamma$. For comparison of the diffusion fields, Fig. 2(b) shows the concentration of $C$ for a phase sequence $\alpha|\beta| \gamma|\alpha| \cdots$. By performing phase-field simulations, the stability of different phase sequences for varying solidification conditions can be investigated. The diffusion processes of the three components are illustrated in a 2D domain of size $200 \times 200$.

Figure 3 shows a time sequence of a 3D simulation of ternary eutectic solidification in a computational domain 60 $\times 90 \times 90$. The computation was initialized with cubic crystal shapes. During the evolution, a regular hexagonal structure of the three isotropic solid phases with $120^{\circ}$ angles between the solid phases is established as steady growth configuration in $3 \mathrm{D}$ in analogy to the lamellar structure in 2D. This symmetry breaks if anisotropy is included.

The simulation in Fig. 4 was conducted with an initial composition vector of $\left(c_{A}, c_{B}, c_{C}\right)=(0.47,0.47,0.06)$ so that the concentration component $c_{C}$ acts as a ternary impurity of minor amount. As can be seen in the first two images, the solid phase $\alpha$ in white color is formed by using up the con- 
centration $c_{A}$ whereas solid phase $\beta$ rejects $A$ atoms. If a $\gamma$ solid phase containing $c_{C}$ as its major composition is introduced, it is instable and immediately dissolves for these concentration proportions. Neither the $\alpha$ phase nor the $\beta$ phase engulfs the concentration $c_{C}$ so that it increases all along the solid-liquid interface. The simulated evolution process recovers the experimentally observed effect that the impurity becomes enriched ahead of the solidifying lamellae and builds up. At larger computational domains, we expect the effect of cell and colony formation to occur.

\section{ACKNOWLEDGMENTS}

The authors gratefully acknowledge the financial support of the German Research Foundation, Grant Nos. Ne 882/1-2, Ne 882/2-2, Ga 695/1-2 and Ga 695/2-2.
[1] I. Steinbach et al., Physica D 94, 135 (1996).

[2] H. Garcke, B. Nestler, and B. Stoth, SIAM J. Appl. Math. 60, 295 (1999).

[3] A. A. Wheeler, G. B. McFadden, and W. J. Boettinger, Proc. R. Soc. London, Ser. A 452, 495 (1996).

[4] J. Tiaden, B. Nestler, H. J. Diepers, and I. Steinbach, Physica D 115, 73 (1998).

[5] Zh. Bi, and R. F. Sekerka, Physica D 261, 95 (1998).

[6] R. Kobayashi, J. A. Warren, and W. C. Carter, Physica D 140, 141 (2000).

[7] M. Plapp and A. Karma, Phys. Rev. Lett. 84, 1740 (2000); Phys. Rev. E 66, 061608 (2002).

[8] B. Nestler and A. A. Wheeler, Phys. Rev. E 57, 2602 (1998); Physica D 138, 114 (2000).

[9] J.-H. Jeong, N. Goldenfeld, and J. A. Dantzig, Phys. Rev. E 64, 041602 (2001).

[10] M. Sabouri-Ghomi, N. Provatas, and M. Grant, Phys. Rev. Lett. 86, 5084 (2001).

[11] W. J. Boettinger, J. A. Warren, C. Beckermann, and A. Karma, Annu. Rev. Mater. Res. 32, 163 (2002).

[12] L. Granasy, T. Borzsonyi, and T. Pusztai, Phys. Rev. Lett. 88, 206105 (2002); J. Cryst. Growth 237-239, 1813 (2002).

[13] M. Apel, B. Boettger, H.-J. Diepers, and I. Steinbach, J. Cryst. Growth 237-239, 154 (2002).

[14] H. Kobayashi, M. Ode, S. G. Kim, W. T. Kim, and T. Suzuki, Scr. Mater. 48, 689 (2003).

[15] J. C. Ramirez, C. Beckermann, A. Karma, and H.-J. Diepers, Phys. Rev. E 69, 051607 (2004).
[16] G. Caginalp, Phys. Rev. A 39, 5887 (1989).

[17] H. Garcke, B. Nestler, and B. Stoth, Physica D 115, 87 (1998).

[18] O. Penrose and P. C. Fife, Physica D 43, 44 (1990).

[19] S.-L. Wang et al., Physica D 69, 189 (1993).

[20] H. Garcke, B. Nestler, and B. Stinner, SIAM J. Appl. Math. 64, 775 (2004).

[21] A. Ahmad, A. A. Wheeler, W. J. Boettinger, and G. B. McFadden, Phys. Rev. E 58, 3436 (1998).

[22] P. Galenko, Phys. Lett. A 287, 190 (2001).

[23] A. Karma and W.-J. Rappel, Phys. Rev. E 53, R3017 (1996); 57, 4323 (1998).

[24] R. F. Almgren, SIAM J. Appl. Math. 59, 2086 (1999).

[25] S. G. Kim, W. T. Kim, and T. Suzuki, Phys. Rev. E 60, 7186 (1999).

[26] G. B. McFadden, A. A. Wheeler, and D. M. Anderson, Physica D 154, 144 (2000).

[27] A. Karma, Phys. Rev. Lett. 87, 115701 (2001).

[28] A. A. Wheeler, W. J. Boettinger, and G. B. McFadden, Phys. Rev. A 45, 7424 (1992).

[29] H. Garcke, R. Haas, and B. Stinner (unpublished).

[30] R. Folch and M. Plapp, Phys. Rev. E 68, 010602 (2003).

[31] S. G. Kim, W. T. Kim, T. Suzuki, and M. Ode, J. Cryst. Growth 261, 135 (2004).

[32] I. Steinbach and F. Pezzolla, Physica D 134, 385 (1999).

[33] C. T. Rios, S. Milenkovic, and R. Caram, Scr. Mater. 48, 1495 (2003).

[34] S. Akamatsu and G. Faivre, Phys. Rev. E 61, 3757 (1999). 